\title{
Survey on Mobile Ad Hoc Network Routing Protocols
}

\author{
Lathigara Amit Maheshbhai \\ PhD Scholar, School of Engineering, \\ RK University, Rajkot, Gujarat, India
}

\author{
K. H. Wandra, Ph.D \\ Director, C. U. Shah College of Engineering \& \\ Technology, Wadhwancity, Gujarat, India
}

\begin{abstract}
Mobile Adhoc Network is described as a network having no physical connections among nodes. Due to nodes mobility, interference, multipath propagation and path loss, MANET is not having any fixed topology. Many routing protocols have been proposed to overcome these characteristics. The purpose of this review paper is to study an existing proactive and reactive routing protocols of MANET depending on their table-driven and on-demand nature respectively. This review paper highlights a summary of these protocols with their functionality, characteristics, benefits and shortcomings along with its performance analysis. Main impartial of this review paper is to make available exploration about enhancement of these existing protocols.
\end{abstract}

\section{Keywords}

MANET, Proactive Routing Protocols, Reactive Routing Protocols

\section{INTRODUCTION}

Mobile Adhoc Network (MANET) [1] is a group of moving nodes that communicates with each other and not consisting of any permanent well defined infrastructure and physical links. For communication, nodes in MANET are accountable for determining other nodes vigorously. MANET is a kind of self-configuring network of mobile nodes associated via wireless links and forms topology randomly. As nodes are mobile in nature and moving freely, randomly and organize indiscriminately thus network's topology may alter quickly and capriciously. Because of it, routing is one of core challenges in MANET. In places where restricted or no communication infrastructures presents, such networks are intended to provide communication capabilities. It is widely used in many military and civilian applications due to such characteristics. MANET is having several significant characteristics like no fixed topologies, restricted bandwidth, energy and physical security. Mainly because of its unique characteristics, wired network's routing protocols cannot be applied straightforward. Possible sample uses of MANET includes students contribution in an cooperative session using laptop, Sharing an information in a conference by business associates, combatants transmitting information for situational cognizance on battle field and workforces synchronizing efforts in emergency disaster assistance later a tornado or tremor. Instead of a stationary network infrastructure, MANET practices multi hop routing to offer network connectivity.

\section{MANET ROUTING PROTOCOL}

Routing protocols in MANET can be categorized primarily as Proactive and Reactive routing protocols [2].

\subsection{Proactive Routing Protocol}

Nodes preserves one or more tables as per necessities, each that comprises up to date information related to routes to any node within network in proactive routing. Each entry comprises subsequent hop for attainment a node and cost of route. Several table driven (proactive) protocols varies in the way the information about a disparity in topology is promulgated through all nodes in network. Among these protocols, there exists some metamorphoses that arises under this group depending on routing information being reorganized in each routing table.

\subsection{Reactive Routing Protocol}

Reactive routing protocols are also recognized as o demand routing protocols because it is not maintaining route information for pair of nodes if there is no communication required. They don't preserves or persistently alter route tables based on up-to-date topology. In case if a node wish to transmit a packet to a different node then this protocol examines for route in an on demand approach and begins connection in command to spread and accept packets. Route discovery typically happened through broadcasting packets named as route request (RREQ) throughout a network.

\section{PROACTIVE ROUTING PROTOCOLS}

Following are few routing protocols, which belongs to proactive approach.

\subsection{Distributed Bellman-Ford (DBF)}

DBF [3] is a proactive protocol based on Bellman-Ford protocol and every node preserves a routing table. Three diverse stages for gathering every required entry are mentioned.

a) Start Conditions: Each node begins with a vector of distances to all nodes of networks attached directly. Each node preserves a routing table with <destination, distance, successor>

b) Send step: Every node sends tuples (destination, distance) as path vector to all direct neighbors. These updates are directing from time to time generally by every second or minute. It depends on the size and the dynamic nature of the network. Quick updates are disseminated whenever destination vectors entries of routing table being changed.

c) Receiving step: For every network $\mathrm{B}$, node discovers shortest distance to A seeing current distance to A and it receipts into account the distance to $\mathrm{A}$ from its neighbors. Node changes its cost to A after doing this for all A destination nodes and then node goes to send step.

\subsection{Destination-Sequenced Distance-Vector Routing (DSDV)}

DSDV [4] is a proactive routing strategy for MANET founded on Bellman-Ford protocol. Fundamental aim of DSDV is to achieve loop-free routing operation. Routing table holds information about a sequence number. (Sequence number is 
usually even in case of existence of link else odd is considered) Destination is producing sequence number and the emitter requires directing out subsequent update with this number. Occasionally routing information is spread between nodes by directing complete dump and slighter incremental updates more repeatedly.

\subsection{Wireless Routing Protocol (WRP)}

WRP [5] is a routing protocol based on distance vector. All node keeps four tables that are for routing, distance, link cost and message retransmission list. MRL (message retransmission list) keeps details like sequence number of update message, retransmission counter and a list of updates sent in update message. Once changes in topology are observed, only path vector tuples (destination, distance) that replicates update are sent. At a time of delivering update messages, each neighbor is essentially required to transmit acknowledgement (ACK) compulsory for each update packet received to advance reliability. If no update messages required to send, WRP exchanges HELLO messages from time to time. If no HELLO message is received in a definite time era, an alarm appears and it has to be verified if the link is reachable still or not. If the node gets a HELLO message from a novel node, the node is added in routing table. WRP sidesteps problem of count-to-infinity by making all nodes to accomplish consistency check of precursor information specified by all its neighbors.

\subsection{Cluster-Head Gateway Switch Routing Protocol (CGSR)}

CGSR [6] uses DSDV as a principal protocol. Mobile nodes are forming clusters and a cluster-head is designated based on distributed algorithm. All nodes inside communication area of cluster-head forms cluster. A gateway node is in the communication area of two or more cluster-heads. Least Cluster Change (LCC) algorithm is using by CGSR to form cluster. Cluster head alteration happens if two cluster-heads appears into one cluster or one of nodes goes out of coverage of all cluster-heads. Cluster-head is capable to regulate a group of nodes, it indicates that it is responsible for forwarding messages, broadcasting within cluster and dynamic channel scheduling. Every node keeps table of cluster member that contains cluster-head for each destination node and routing table based on distance vector that contains succeeding hop for destination. Cluster member table is broadcasted from time to time. A node will reflect entries in its table of cluster member on receiving a new one from its neighbors.

\subsection{Global State Routing (GSR)}

GSR [7] is centered on Link State routing (LS). GSR does not flood link-state packets like LS. Instead, every node keeps its link state table updated. It will exchange its LS information from time to time with its neighbors merely. It means that GSR is medium access control layer proficient as it preserves the control message overhead low. GSR still discovers optimal and precise paths. GSR could be designated as being grounded on LS routing, which has the benefit of routing accurateness and the broadcasting method used in DBF to circumvent ineffective flooding like in LS routing. Each node keeps topology table, neighbor list, distance table and next hop table.

\subsection{Optimized Link State Routing Protocol (OLSR)}

Every node in OLSR [8] broadcast "Hello" message from time to time with facts to particular nodes of network to interchange neighborhood details. It contains sequence number, a list of distance info of node's neighbors and IP of node. In receipt of this info a node constructs itself a routing table. Now node can determine route to every node it wants to communicate with shortest path algorithm. Upon receiving information packet, node checks sequence number and if it is duplicate then node will discard it. For each node within the network, it preserves route information. The information is updated only if detects any alteration in neighborhood or route to any destination is terminated or a superior route is identified for a destination

\subsection{Fisheye State Routing (FSR)}

FSR [9] is a proactive routing protocol with implicit hierarchical routing structure. Link-state info changes with diverse rates as per range of fisheye. FSR works effectively to large network by preserving low overhead without negotiating route calculation accurateness when destination is nearby. FSR's routing precision is similar with a model link-state structure. FSR escapes extra work of searching destination by holding a routing entry for each destination and preserves low latency of particular packet transmission. If mobility increases, remote destinations route becomes inaccurate. However, when a packet reaches its destination, it discovers ever more precise routing instructions as it enters in sectors with a greater refresh rate. Due to it, FSR is further appropriate for huge mobile networks with high mobility and restricted bandwidth. Under appropriate radius size along with number of scope levels, FSR shows to be a flexible way out to challenge of keeping correct routes in ad-hoc networks.

\subsection{Hierarchical state routing (HSR)}

HSR [10] preserves an ordered topology where lowest level cluster-heads turn out to be members of succeeding higher level. Super clusters are formed on upper level and so on. If node wishes to interconnect to a node of outside's cluster then it is required to request own cluster-head to advancing packet to subsequent level, until it reaches to a preferred destination node's cluster-head. Further packet moves down to destination node. Instead of geological way, HSR recommends logical way for cluster nodes: nodes within identical groups or in the identical battle group are clustered together, assuming they will communicate considerably within logical cluster.

\subsection{Source Tree Adaptive Routing protocol (STAR)}

STAR [11] works with link-state information and it was first proactive routing algorithm. It is quicker than on-demand algorithms. It is a first table-driven protocol where LORA standard is applied. To keep control messages low, it doesn't takes shortest path. Every node is identified with a fix address. Immense benefit is that no periodical updates are required. Source tree holds links to all neighbor after start procedure. During update stage, STAR directs own source tree to all other neighbors as update instantly. So every node can constructed with its own source tree and received ones, a topology graph comprising whole network. Such updates comprise of one or additional Link-State update unit. 


\subsection{Topology Broadcast based on Reverse- Path Forwarding (TBRPF)}

TBRPF [12] practices the theory of reverse-path forwarding to broadcast each link-state update in reverse way along the spanning tree calculated by smallest hop route from all nodes to the source of update. Each link state update is broadcast along the minimum hop path tree rooted at the source of update. The broadcast trees are reflected animatedly using the received topology information along the trees themselves, thus necessitating very slight further overhead for preserving the trees. Minimum hop path trees are used since they alters not as much of repeatedly than shortest path trees created on a metric like delay. Based on the received information, each node calculates its parent and children for the broadcast tree directed at each source. Each node forward updates initiating from source to its children on the tree directed at source. Using sequence numbers, TBRPF accomplishes reliability despite of changes in topology.

\subsection{Intrazone Routing Protocol (IARP)}

IARP [13] is founded on Link State protocol. Node observes its particular link state, means their local neighbors, with neighbor discovery protocol. It provides existing information about node's neighbors such as address. A packet with fresh information sending by each node to its direct neighbors from time to time. With fresh information received, nodes calculate their information further and updates routing table. TTL (time to live) count is available in original info packet. TTL is prepared by node and transmit in packet with new information as $r-1$ hops where $r$ considered as zone radius. Once node receives packet, it's required to update routing table and TTL value is decremented. Once value of TTL becomes zero, packet is discarded. Packet with local information halts in its own zone and no other zone can receive this information.

\subsection{Better Approach to Mobile Adhoc Networking (BATMAN)}

BATMAN [14] is a table driven kind of MANET's routing protocol. It proactively preserves information about presence of all nodes that are reachable through single or multi hop communication links in mesh. The stratagem of BATMAN is to discover for each target in mesh one single hop neighbor that can be further used as finest gateway to communicate with target node. For multi hop routing based on IP, routing table of a node must encompass a link local gateway for each network path or node. Every node directs "broadcast" for time to time, by this means notifying about its presence to all its neighbors. Further neighbors directs same to their neighbors. It conveys information to all nodes within a network. For finding a suitable path to a definite node, BATMAN reckonings accepted originator-messages and records about neighbor, from whom message derived not identical to link state protocols but same as distance vector protocols. It is not trying to elect the complete route but by using messages from originator, first step in the right route. Further data is passed over to succeeding neighbor node in same direction, who in turn uses the identical method. It replications up until data conveys to target node.

\subsection{BABEL}

BABEL [15] is a kind of innovative distance vector table driven routing protocol. It is new-fangled than BATMAN and OLSR but drolly, its scheme is grounded on protocol like DSDV. Sequence number is using to eradicate count to infinity problem alike DSDV. EIGRP's loop anticipation techniques using viability circumstances adopted by BABEL to hastily converge on loop free routes. ETX metric is used by BABEL alike OLSR. BABEL updates are communicated untrustworthily using IP6. Within sparse networks, BABEL can outperform among contending routing algorithms.

\section{REACTIVE ROUTING PROTOCOLS}

This section highlights routing protocols that belongs to reactive (on demand) approach.

\subsection{Dynamic Source Routing (DSR)}

DSR [16] is a proficient and simple protocol intended precisely for use in MANET. It permits network to be utterly self-configuring and self-organizing without any necessity for prevailing network administration or setup. Route discovery and Route maintenance are two focal procedures of DSR that works collectively to countenance nodes to determine and sustain paths to haphazard destinations in network. All features of DSR runs on demand exclusively that further countenances routing packet overhead of DSR to scale inevitably to only what is obligatory to counter alteration in active routes. DSR consents numerous paths to specific target node and permits each sender node to manage and select the paths used in its packets routing.

\subsection{Ad hoc On-Demand Distance Vector (AODV)}

AODV [17] is anticipated to use on-demand in mobile adhoc network. AODV deals nippy and effectively against deviation in vigorous link disorders, stumpy network consumption, overhead in process and memory with discovery of unicast paths to target nodes within network. Loop free operation seamlessly provided by sequence numbers provided by destination nodes and avoid problems like "count to infinity" found in classical protocols based on distance-vector.

\subsection{Temporally-Ordered Routing Algorithm (TORA)}

TORA [18] is a kind of routing protocol in MANET that works in distributed manner. Within an autonomous system, its envisioned usage is for routing of internet protocol datagrams. Fundamentally TORA is neither a link-state nor a distance-vector but it is a kind of algorithm referred as link reversal. Preliminary it organizes network through diffusing computations of temporally ordered sequence where individually computation comprising of a sequence of directed link reversals. TORA is exceedingly effective, adaptive, scalable and well-suited for dense and huge networks of mobile nodes where its performance on failure of link is archetypally comprises of localized single-pass of the distributed algorithm only. It is accomplished with practice of either logical or physical clock that creates topological change events for temporal order.

\subsection{Cluster Based Routing (CBR)}

Nodes within mobile ad hoc network are grouped together in a distributed style into numerous coinciding or dismember two hop breadth clusters and to preserve information related to cluster membership, a cluster head is nominated for every clusters [19]. Based on information related to cluster membership retained at each cluster head, routes among inter cluster are vigorously discovered. CBR adroitly diminishes flooding traffic of route discovery by grouping nodes into clusters and improves entire process. DSR uses unidirectional links for both inter and intra cluster routing. 


\subsection{Associativity Based long lived Routing (ABR)}

ABR [20] is considered as bandwidth proficient distributed routing protocol that doesn't endeavor to regularly preserve information related to routing for all nodes. As nodes are acting as a router in MANET and due to mobility of nodes routes are keep changing. ABR chooses route based on associativity states belongs to node that suggest connection period, temporal, spatial and signal stability. Based on it selected route is probable to be enduring and hence there is no necessity to start again recurrently. Ultimately it results into higher attainable throughput. ABR is established on reactive source initiated strategy. Based on necessity, route requests are initiated. Quick-abort strategy and localized-query are correspondingly combined into $\mathrm{ABR}$ to advance route unearthing time during ruin of association property.

\subsection{Relative Distance Micro-discovery Ad Hoc Routing (RDMAR)}

RDMAR [21] is scalable, bandwidth efficient and highly adaptive on demand protocol. Its retort in contradiction of link failure is stereotypically localized to a precisely lesser area in network close to change is one of key ideas in its design. Relative Distance Micro-discovery (RDM) is introduced as mechanism to determine a route to achieve said behavior. RDM's main concept is about localizing a query flood by knowing a kin distance (also known as relative distance - RD) among two nodes. To achieve this, all time a route discovery among two nodes is prompted an iterative procedure that determines a guesstimate of their kin distance, provided a mediocre mobility of nodes and historical information ever since they former communicated along with their preceding kin distance. Query flood is further localized within a restricted area in a network centered at source node of route discovery based on the recently considered kin distance and with maximum propagation radius that matches to expectable kin distance. Specified capability to localize query flooding within a limited area in a network further improves scalability, effectively diminish overhead incurred for routing and controls overall network congestion.

\subsection{Location-Aided Routing (LAR)}

LAR [22] exclusively uses information related to location of mobile nodes to reduce control overhead associated with discovery of route among nodes. Global positioning system is used to obtain information about location of mobile nodes. LAR uses zones like Expected and Requested. Expected zone is area that source may assumes that destination may contained at particular time $\mathrm{T}$ estimated by source. Request zone is area within which nodes are allowed to forward route request. Two LAR schemes are available to discover route efficiently within restricted search area that also results in to low control overhead.

\subsection{Ad hoc On-demand Multipath Distance Vector (AOMDV)}

AOMDV [23] is able to search numerous paths among nodes at time discovery of route. AOMDV intended to work mostly in exceedingly dynamic mobile ad hoc network where failure of links and further routes arises repeatedly. Protocols that works with single path among nodes like AODV requires fresh discovery of route in case of link failure in active route. High control overhead and latency as well concomitant with every single discovery of route. Through readiness of multiple redundant paths among nodes, mentioned inadequacy can be evaded. AOMDV entails a fresh discovery of route only when all routes to destination breaks. AOMDV involves pintsized supplementary overhead for establishment of multiple paths as it is using information associated to routing already existing in principal AODV as ample as conceivable.

\subsection{Flow State in the Dynamic Source Routing (FSDSR)}

FSDSR [24] is considered as an encroachment to DSR. It is a proficient and simple routing protocol intended to use precisely for MANET. All features of FSDSR functions ondemand exclusively. DSR facilitates source of a packet to describe the nodes sequence that packet must shadow to reach at target and to route that packet beside with hop sequence by comprising a source route header within a packet. It tolerates routing overhead of DSR to scale spontaneously to only that required to react to changes in active routes. "flow-state" is reflected as an extension of DSR that permits the routing of most packets without a source route header within a packet explicitly. Even conserving ultimate properties of DSR, still FSDSR reduces control overhead excellently.

\subsection{Dynamic NIx-Vector Routing (DNVR)}

DNVR [25] guarantees a loop freedom operation and preserves routes based on request alike other reactive routing protocols. DNVR has a number of miscellaneous features that makes it diverse from other reactive routing protocols like depletion of probes for proficient recognizing of network, authentication of kept route information, conquest of route requests for a traditionalist discovery of route, routing states administration in a well-timed manner, address resolution exclusion and compact form of source routes usage.

\subsection{Reliable Ad-hoc On-demand Distance Vector routing (RAODV)}

RAODV [26] disentangles the problem of an attack by selfish and malicious nodes using co-operative security slant based on local monitoring. RAODV is a tactic to integrate reliability level of nodes into traditional routing metrics to discover route. RAODV is ashore on AODV with a postulation that nodes cannot satirize and all relevant conditions of network are upright. RAODV acts as good as AODV in absenteeism of an attack and perceives and insulates nodes that are misbehaving in incidence of an attack. RAODV recuperates from an attack once a misbehaving node goes out from network or turn out to be virtuous. CORE and CONFIDANT kind of distinctive hardware are no longer essential in RAODV.

\subsection{Dynamic MANET On demand (DYMO)}

DYMO [27] protocol allows multihop unicast reactive routing between contributing DYMO routers. Route discovery and maintenance are considered as basic operations of DYMO. Route Request (RREQ) packet is broadcasting by originator's DYMO router all over the network to discover a path to target's DYMO router during route discovery. Each transitional DYMO router keeps a note of a route to the originator DYMO router during this hop by hop broadcasting procedure. When target's DYMO router obtains first RREQ, it replies with a Route Reply (RREP) to the originator. Each transitional DYMO router that obtains RREP makes a route to the target, and then the RREP is unicast hop by hop toward originator. Once originator's DYMO router obtains RREP, routes have then been recognized between originating and target DYMO router in both directions. Two main operations are in route maintenance. To preserve routes in usage, 
DYMO routers extends lifetime of route upon fruitfully dispatching a packet. In case of change in network topology, DYMO routers observes routes where traffic is rolling. When a data packet is received for dispatching and a route for destination is not recognized or a route is damaged, then DYMO router of the originator of the packet is reported. A Route Error (RERR) is directed toward originator to intimate that the route to particular target is either missing or invalid. When the originator's DYMO router obtains RERR, it simply obliterates the route. If this originator's DYMO router obtains a packet for the same destination later, it will essential to accomplish route discovery another time for that destination. Sequence numbers are using in DYMO for loop freedom assurance.

\subsection{Admission Control enabled On- demand Routing (ACOR)}

ACOR [28] is a reactive kind of routing protocol intended to make available Quality-of-Service (QoS) among end-to-end route. ACOR uses simple local cost and global cost functions to represent at each node and at the end-to-end cost of a route respectively, inherent resource reservation amended along with simple admission control strategy against consistent change of network topology.

\section{CONCLUSION}

In this review paper, several existing MANET's routing protocols are described. Classifications of routing protocols are specified as proactive and reactive. Every nodes are preserving conversant routing information to all remaining nodes within network in proactive protocols. A node is required to quest a route to a destination once transmission of packet is required for same destination. Numerous proactive protocols are pondered like DBF, DSDV, WRP, CGSR, GSR, OLSR, FSR, HSR, STAR, TBRPF, IARP, BATMAN and BABEL. Destination sequence number is used in DSDV and GSR to retain conversant loop-free paths. HSR also have its place in cluster of hierarchical protocols. For nodes resides further away, FSR diminishes size of tables needed to be bartered by preserving a smaller amount of precise information. Nodes may be congregated together to form clusters and CGSR is a kind of cluster-based protocol. BABEL speeds up convergence by reactively requesting a new sequence number. BATMAN also keep routes loop-free. Several reactive protocols are discussed like DSR, AODV, TORA, CBR, ABR, RDMAR, LAR, AOMDV, DNVR, RAODV, DYMO and ACOR. LAR and RDMR have identical cost as traditional flooding algorithm in worst-case scenario. Stable routes are selected in ABR by destination using node stability information. Likewise it permits shortest route selection by destination node. It may perform better than DSR and connection setup delay is lower in AODV.

\section{ACKNOWLEDGMENTS}

The authors would like to show gratitude the reviewers for their fruitful recommendations and intuitive remarks that have facilitated us to deepen the content and improvement in presentation of this paper.

\section{REFERENCES}

[1] S. Corson and J. Macker,"Mobile Ad hoc Networking (MANET): Routing Protocol Performance Issues and Evaluation Considerations," RFC 2501, Jan. 1999

[2] E. M. Royer and C.-K. Toh,"A Review of Current Routing Protocols for Ad-Hoc Mobile Wireless
Networks", IEEE Personal Communications Magazine, April 1999, pp. 46-55

[3] D. Bertsekas and R. Gallager, "Data Networks" Prentice Hall Publ., New Jersey, 2002.

[4] C. E. Perkins and P. Bhagwat, "Highly dynamic destination-sequenced distance vector routing (DSDV) for mobile computers", Proceedings of ACM SIGCOMM 94, 1994, pp. 34-244.

[5] S. Murthy and J. J. Garcia-Luna-Aceves, "An Efficient Routing Protocol for Wireless Networks", ACM Mobile Networks and App. Journal, Special Issue on Routing in Mobile Communication Networks, pp.183-97, 1996.

[6] C.-C. Chiang, Routing in clustered multihop mobile wireless networks with fading channel, in: Proceedings of IEEE SICON, April 1997, pp. 197-211.

[7] Tsu-Wei Chen and M. Gerla, "Global State Routing: A New Routing Scheme for Ad-hoc Wireless Networks" Proceedings of International Computing Conference IEEE ICC 1998.

[8] P. Jacquet, P. Muhlethaler, T. Clausen, A. Laouiti, A. Qayyum, L. Viennot," Optimized link state routing protocol for ad hoc networks", IEEE INMIC, Pakistan, 2001.

[9] M. Gerla, Fisheye state routing protocol (FSR) for ad hoc networks, Internet Draft, draft-ietf manet-aodv-03.txt, work in progress, 2002.

[10] G. Pei, M. Gerla, X. Hong, C. Chiang, "A wireless hierarchical routing protocol with group mobility", in: Proceedings of Wireless Communications and Networking, New Orleans, 1999.

[11] J.J. Garcia-Luna-Aceves, C. Marcelo Spohn, "Sourcetree routing in wireless networks", in: Proceedings of the Seventh Annual International Conference on Network Protocols Toronto, Canada, October 1999, p. 273.

[12] B. Bellur, R.G. Ogier, F.L Templin, "Topology broadcast based on reverse-path forwarding routing protocol (tbrpf)", in: Internet Draft, draft-ietf-manet-tbrpf-06.txt, work in progress, 2003.

[13] Zygmunt J. Haas, Marc R. Pearlman, Prince Samar, "The Intrazone Routing Protocol (IARP) for Ad $\mathrm{Hoc}$ Networks", draft-ietf-manet-zone-iarp-00.txt

[14] A. Neumann, C. Aichele, M. Lindner, S. Wunderlich, "Better Approach To Mobile Ad-hoc Networking (BATMAN)" draft-wunderlich-openmesh-manetrouting-00.txt

[15] Juliusz Chroboczek, "The Babel Routing Protocol", Internet-Draft, April 2009.

[16] D. B. Johnson and D. A. Maltz, "Dynamic Source Routing in Ad-Hoc Wireless Networks," Mobile Computing, T. Imielinski and H. Korth, Eds., Kluwer, 1996, pp. $153-81$

[17] S. Das, C. Perkins, E. Royer, Ad hoc on demand distance vector (AODV) routing, Internet Draft, draft-ietfmanetaodv-11.txt, 2002

[18] V.D. Park, M.S. Corson, A highly adaptive distributed routing algorithm for mobile wireless networks, in: Proceedings of INFOCOM, April 1997 
[19] M. Jiang, J. Ji, Y.C. Tay, Cluster based routing protocol, Internet Draft, draft-ietf-manet-cbrp-spec-01.txt , 1999.

[20] C. Toh, A novel distributed routing protocol to support ad-hoc mobile computing, in: IEEE 15th Annual International Phoenix Conf., 1996, pp. 480-486

[21] G. Aggelou, R. Tafazolli, RDMAR: a bandwidthefficient routing protocol for mobile ad hoc networks, in: ACM International Workshop on Wireless Mobile Multimedia (WoWMoM), 1999, pp. 26-33

[22] Y.-B. Ko, N.H. Vaidya, Location-aided routing (LAR) in mobile ad hoc networks, in: Proceedings of the Fourth Annual ACM/IEEE International Conference on Mobile Computing and Networking (Mobicom_98), Dallas, TX, 1998

[23] Mahesh K. Marina, Samir R. Das,"On-demand Multipath Distance Vector Routing in Ad hoc Networks",IEEE 2001
[24] Yih-Chun Hu, David B. Johnson, David A. Maltz, Flow State in the Dynamic Source Routing Protocol for Mobile Ad Hoc Networks, Internet Draft, draft-ietfmanet-dsrflow-00.txt

[25] Young J. Lee and George F. Riley, Dynamic NIx-Vector Routing for Mobile Ad Hoc Networks. Proceedings of the IEEE Wireless Communications and Networking Conference (WCNC 2005), New Orleans, Mar. 13 - 17, 2005

[26] Sandhya Khurana, Neelima Gupta, Nagender Aneja, Reliable Ad-hoc On-demand Distance Vector Routing Protocol, Proceedings of the Fifth International Conference on Networking (ICN 2006)

[27] I. Chakeres, Dynamic MANET On-demand Routing Protocol, Internet Draft, draft-ietf-manet-dymo-00.txt

[28] N. Kettaf, Admission Control enabled On demand Routing (ACOR), Internet Draft, draft-kettaf-manet-acor03.txt 